\title{
Variabilidad y tendencia de la temperatura superficial de los grandes embalses del Río Negro
}

\section{Surface water temperature trend and variability of the Negro River large reservoirs}

\author{
Manta, Gastón (1); Alcántara, Ignacio $(2,3)$ \\ (1) Departamento de Ciencias de la Atmósfera, Facultad de Ciencias, Universidad de la República, Montevideo, Uruguay. \\ (2) Departamento de Microbiología, Instituto de Investigaciones Biológicas Clemente Estable, Montevideo, Uruguay. \\ (3) Departamento de Bioestadística, Facultad de Veterinaria, Universidad de la República, Montevideo, Uruguay. \\ Contacto: gmanta@fisica.edu.uy
}

RECIBIDO: 4/7/2018 APROBADO: 4/10/2018

\begin{abstract}
Resumen
La temperatura del agua es un parámetro fundamental para comprender la dinámica de los cuerpos de agua continentales. Se estudió la variabilidad y tendencia de la temperatura superficial de los grandes embalses del Río Negro, Rincón del Bonete y Baygorria, utilizando 16 años de datos diarios satelitales (MUR-GHRSST) con resolución espacial de $0,01^{\circ}$ entre 2002 y 2018 . La temperatura media fue de $18,8^{\circ} \mathrm{C}$ y julio (enero) el mes más frío (cálido) con un promedio de $12,1^{\circ} \mathrm{C}\left(25,2^{\circ} \mathrm{C}\right)$. Mientras que otoño y primavera presentan la mayor variabilidad intraestacional, invierno presenta la mayor variabilidad interanual. La temperatura superficial del sistema mostró una tendencia significativa de aumento de $1,3{ }^{\circ} \mathrm{C}$ por década para primavera-verano, mientras que las estaciones de otoño e invierno no presentaron tendencia significativa. Se observó a su vez en el eje espacial una tendencia al aumento de la temperatura desde aguas arriba hacia aguas abajo. La información satelital fue correlacionada con mediciones in situ de oportunidad $(\mathrm{n}=67)$ y se obtuvo una correlación de 0,94 y un error cuadrático medio de $1,92^{\circ} \mathrm{C}$. Generar series temporales de mediciones in situ dirigidas permitiría una evaluación del producto y consolidar su uso operativo. Los resultados demuestran la utilidad del sensoramiento remoto de la temperatura del agua en sistemas continentales como herramienta de monitoreo basado en información de libre acceso. Además, se describe por primera vez el ciclo estacional, la variabilidad y tendencia de los embalses del Río Negro, esperando que los resultados aporten para las medidas de manejo ambiental.

Palabras clave: Climatología, Rincón del Bonete, Baygorria, sensoramiento remoto, calentamiento aguas superficiales.
\end{abstract}

\begin{abstract}
Water temperature is a fundamental parameter to understand the dynamics of continental waters. Surface water temperature trend and variability of the large reservoirs of the Negro River, Rincón del Bonete and Baygorria, using 16 years of satellite data (MUR-GHRSST) with a spatial resolution of $0.01^{\circ}$ between 2002 and 2018 was studied. The average temperature was $18.8^{\circ} \mathrm{C}$, with a minimum of $12.1{ }^{\circ} \mathrm{C}$ and maximum of $25.2{ }^{\circ} \mathrm{C}$, in July and January, respectively. Spring and autumn showed the highest intraseasonal variability, and winter the highest interannual variability. The surface temperature of the reservoirs showed a significant trend towards warming of $1.3^{\circ} \mathrm{C}$ per decade for spring-summer, while the seasons of autumn and winter did not exhibited a significant trend. The warming trend increased towards downstream. The satellite information was correlated with opportunistic observed data $(n=67)$, with a correlation of 0.94 and a root mean square error of $1.92{ }^{\circ} \mathrm{C}$. Generating time series of continuous measurements in situ would allow an evaluation of the product and viability for operational usage. These results demonstrate the utility of remote sensing of water temperature as a monitoring tool based on freely accessible information. In addition, the seasonal cycle, trend and variability of the reservoirs of the Río Negro were described for the first time. It is expected that these results contribute to the environmental management measures of the Negro River reservoirs. Keywords: Climatology, Rincón del Bonete, Baygorria, Remote Sensing, Surface Water Warming.
\end{abstract}

\section{Introducción}

En los sistemas lóticos (principalmente ríos y arroyos), la temperatura del agua varía a escalas de tiempo diarias, estacionales, interanuales, y a lo largo del eje longitudinal del cauce (Webb, et al., 2008). También se produce una heterogeneidad térmica considerable a escalas de meso y micro-hábitat en respuesta a la temperatura del aire local y la velocidad del viento, que influyen en el enfriamiento por evaporación y la extensión de la sombra topográfica y ribereña 
(Poole y Berman, 2001). En los cuerpos de agua lénticos (en especial lagos y embalses), las aguas superficiales se calientan principalmente por acción de la radiación proveniente del sol y pierden densidad. Esto tiene como consecuencia un aumento en la resistencia térmica a la mezcla de las capas de agua superficiales con respecto a las sub-superficiales (Hambright, et al., 1994). Una diferencia de solo unos pocos grados es suficiente para evitar la circulación completa entre superficie y fondo, condición denominada estratificación térmica (Hutchinson, 1941). Otros fenómenos físicos tales como el viento o corrientes producen turbulencias que favorecen la mezcla de la columna de agua, particularmente en cuerpos de agua poco profundos (Tundisi, et al., 2004). En los casos de sistemas mixtos (ríos y embalses) existe una tendencia al aumento de la temperatura aguas abajo de las represas o embalses. Esto se debe a que el detenimiento del cauce incrementa el tiempo de residencia del agua y por lo tanto la superficie expuesta a la radiación solar es mayor, lo que permite un mayor calentamiento (Allan y Castillo, 2007).

La temperatura es un factor regulador del metabolismo de los organismos (Brown, et al., 2004). El aumento de la temperatura hasta un límite tolerable produce un incremento en las tasas de producción primaria y de respiración por parte de autótrofos y heterótrofos (Straile, 2005; Reynolds, 2006). A su vez, la temperatura también tiene efectos indirectos en los procesos físicos, resultando en gradientes de calor característicos que a su vez son responsables de gradientes químicos y de la distribución de organismos (Poole y Berman, 2001). En el caso de los microorganismos que viven en suspensión de la columna de agua (plancton), la estratificación térmica impone una barrera para su capacidad de migración vertical (Margalef, 1983). Algunas especies de cianobacterias con capacidad de regulación de la flotabilidad pueden posicionarse en profundidades favorables y dominar a otras especies del fitoplancton como los unicelulares y coloniales no flotantes (Reynolds, et al., 1987; Joehnk, et al., 2008).

El Río Negro es uno de los ríos más importantes de Uruguay. Presenta una amplia extensión de $810 \mathrm{~km}$ desde sus nacientes en Brasil a su desembocadura en el río Uruguay. Su cuenca de drenaje abarca aproximadamente $71.400 \mathrm{~km}^{2}$, de los cuales casi $69.000 \mathrm{~km}^{2}$ le corresponden a Uruguay (Pérez, 2002). Cuenta con tres represas en cadena construidas para obtención de energía eléctrica: Rincón del Bonete $\left(1070 \mathrm{~km}^{2}\right)$, Rincón de Baygorria $\left(100 \mathrm{~km}^{2}\right)$ y Palmar $\left(320 \mathrm{~km}^{2}\right.$; Chalar, et al., 2014) (Figura 1). El uso predominante de los suelos de la cuenca es la ganadería extensiva (con pradera mejorada). Se destaca también el aumento reciente de agricultura intensiva y forestación (Chalar, et al., 2014; MGAP, 2017). El río es considerado como eutrófico (Chalar, et al., 2014; MVOTMA, 2018) y presenta frecuentemente floraciones de cianobacterias de los géneros Microcystis y Dolichospermum, especialmente en sus embalses durante los meses de verano (González-Piana, et al., 2011; Chalar, et al., 2014). Estudios recientes reportan concentraciones de cianotoxinas (Microcistinas) en niveles de riesgo moderado para el uso recreacional del río (González-Piana, et al., 2017); e inclusive el embalse de Baygorria cuenta con el registro más alto de microcistinas (38 mg. $\mathrm{L}^{-1}$ ) para el país (Bonilla, et al., 2015).

Si bien existen antecedentes de campañas de monitoreos sistemáticos de parámetros fisicoquímicos y biológicos in situ (ej. Chalar, et al., 2015; González-Piana, et al., 2017, DINAMA, 2018), las series temporales de temperatura por satélite no han sido aún exploradas para los embalses del Río Negro. El uso de datos satelitales presenta la ventaja de tener una amplia cobertura espacio-temporal, lo cual permite evaluar la evolución del fenómeno en el tiempo. La principal desventaja es que se obtiene únicamente el dato de superficie, por lo que no sustituye las mediciones de perfiles in situ (Martin, et al., 2012).

Una lista de los instrumentos y técnicas empleadas clásicamente durante las primeras décadas de medición remota de temperatura superficial del agua (TSA) se encuentra resumida en Barton (1995). Los desafíos que presenta el uso de esta herramienta en ambientes continentales y costeros es la contaminación del dato (píxel), debido a que, principalmente por la resolución horizontal del sensor y la morfología de la costa, queda incluida tanto la tierra como el agua en el píxel (Martin, et al., 2012; Chang, et al., 2015). Debido a este problema, algunas de las primeras bases de datos de TSA de lagos, medidas a través de satélites y utilizando el producto AVHRR implementado por NASA/NOAA desde principios de 1970, solo tomaban en cuenta grandes cuerpos de agua ampliamente mayores a $10 \mathrm{~km}$ de diámetro (Sharma, et al., 2015). A principios de la década del 2000, el Experimento Global de Asimilación de Datos Oceánicos (GODAE) proporcionó un proyecto piloto de mediciones de TSA de alta resolución aquí utilizado, el cual se compone de una combinación ("blended") de varios productos de datos TSA, que incluye diferentes sensores satelitales y observaciones in situ, con medidas en tiempo real (Chang, et al., 2015; Dash, et al., 2012). Con esta nueva herramienta, estudios más recientes sugieren, a partir de la correlación con datos in situ, que a partir de $10 \mathrm{~km}$ de diámetro del cuerpo de agua se obtiene buena calidad de mediciones (Crosman, et al., 2017). A su vez, resaltan que la principal dificultad se encuentra en zonas de altas latitudes y regiones más nubosas (Crosman, et al., 2017). Esta herramienta ha sido implementada en Kraemer et al. (2017), donde estudiaron la relación entre la TSA obtenida del producto aquí utilizado y la Clorofila- $a$ (obtenida mediante MODIS) en 188 lagos de todo el mundo cuyas áreas fueran mayores a $50 \mathrm{~km}^{2}$.

La motivación de este estudio es caracterizar la variabilidad y tendencia de la temperatura de superficie del Río Negro utilizando mediciones satelitales de TSA y evaluar las oportunidades e implicancias en el manejo de los grandes embalses del Río Negro.

\section{Materiales y Métodos}

Se trabajó con datos diarios satelitales de TSA procesados por el "Grupo de alta resolución de temperatura superficial del mar" (GHRSST por su sigla en inglés, https://www.ghrsst. org/) (Martin, et al., 2012). Estos datos se encuentran disponibles para descargar libremente en la página web del Centro de Archivo Activo de Distribución de Datos en Oceanografía Física (podaac) del Laboratorio de Propulsión a Chorro de la Administración Nacional de Aeronáutica y el Espacio de Estados Unidos (NASA; https://podaac.jpl.nasa.gov/) y también se pueden observar en tiempo real en varios sitios web (por ejemplo: https://podaac-tools.jpl.nasa.gov/soto/) La información se presenta grillada y sin datos faltantes (nivel de procesamiento 4), de modo que en cada píxel asignado como agua siempre hay un dato. Aunque en algún momento, ya sea por descenso del nivel del cuerpo de agua o por nubes, no sea posible su medición, este es interpolado estadísticamente. Este producto cuenta también con una variable llamada análisis de error, que es el desvío estándar del error estimado de la 
medición y que se utilizó para encontrar diferencias espaciales en la precisión del dato.

El GHRSST produce este conjunto de datos en tiempo real (latencia de un día) y en retrospectiva con mayor control de calidad (latencia de cuatro días), utilizando "wavelets" como funciones base en un enfoque de interpolación óptima en una grilla global de $0,01^{\circ}$ (cerca de $1 \mathrm{~km}$ para latitudes medias). La versión utilizada en este trabajo es la "Multiscale Ultrahigh Resolution" (MUR). Esta versión se basa en observaciones nocturnas de los primeros milímetros del espejo de agua de varios instrumentos, incluido el Advanced Radiometer Scanning-EOS de la NASA (AMSRE), el espectrorradiómetro de imágenes de resolución moderada (MODIS Aqua y Terra) de la NASA, el radiómetro WindSat de microondas de la Armada de los EE.UU., el Radiómetro Avanzado de Muy Alta Resolución (AVHRR) y varios satélites NOAA de órbita polar, así como observaciones in situ del proyecto NOAA iQuam (Martin, et al., 2012).

Los conjuntos de datos de TSA basados en satélites tienen resoluciones drásticamente diferentes. Por ejemplo, el sensor de tipo infrarrojo puede tener una resolución muy alta de hasta $1 \mathrm{~km}$ en distancia horizontal, pero los datos del sensor de microonda suelen tener una resolución menor, cercana a 25 $\mathrm{km}$. Todos los datos son generalmente muestreados en patrones irregulares bajo las órbitas de los satélites. Además de todo esto, hay muchos vacíos de datos (agujeros en la cobertura de datos) debido a contaminaciones por nubes, aerosoles y tierra. Para tratar objetivamente estos problemas de muestreo MUR utiliza una técnica llamada Análisis Variacional de Resolución Múltiple (MRVA). MRVA es un método de interpolación estadística basado en la descomposición wavelet, llamado "análisis de resolución múltiple", cuya virtud es que el mapa de TSA se puede controlar sin degradar la representatividad local. La expresión matemática de la descomposición wavelet se encuentra en Chin et al. (1998). La principal ventaja de MUR es que al momento es el único análisis diario global de TSA disponible libremente en tiempo real con resolución horizontal de $0,01^{\circ}$ (Crosman, et al., 2017).

El período de estudio analizado abarca desde que se encuentran los datos disponibles (1 de junio de 2002 hasta el 31 de mayo de 2018), hasta completar un total de 16 años. Se contó con 5845 días con datos en 1513 pixeles, totalizando un área de cobertura de $1513 \mathrm{~km}^{2}$. La climatología diaria se construyó haciendo el promedio para cada día del año y un suavizado con una media móvil centrada de 30 días. También se realizó un análisis espectral de la serie temporal utilizando el método de Lomb (Press, et al., 1992). Se trabajó con la media anual y medias estacionales; se consideran verano los meses de diciembre-enero-febrero, otoño: marzo, abril y mayo, invierno: junio, julio y agosto, y primavera: setiembre, octubre y noviembre. La variabilidad se estimó a partir del desvío estándar de la temperatura calculado para cada día del año promediado espacialmente, y luego distinguiendo a nivel espacial para cada estación del año la componente climatológica (la variabilidad promedio dentro de cada estación) y la componente interanual (la variabilidad año a año de cada estación).

Para estudiar si los embalses del Río Negro tienden a aumentar o disminuir su temperatura, se realizó un análisis de tendencia ajustando un modelo de regresión lineal para la temperatura media para todo el año y a su vez para cada estación, utilizando como variable explicativa el paso del tiempo, siempre sobre el promedio anual $(n=16)$, ya sea para cada estación o para todo el año. La significancia estadística se evaluó aplicando el test de Student con nivel de confianza del 95\%. En el caso del análisis espacial se graficó la pendiente del ajuste de la recta para cada píxel solo donde se encontró significancia estadística. Los mapas se presentan en líneas de contornos a los efectos de facilitar la visualización, ya que el producto de nivel 4 contiene un suavizado y sin datos fuera de rango (Martin, et al., 2012).

Para la comparación de los datos satelitales con observaciones in situ se utilizaron dos bases de datos. La primera, correspondiente a información disponible de forma libre del observatorio ambiental nacional de la Dirección Nacional de Medio Ambiente (DINAMA, 2018). Se utilizaron los datos correspondientes a la estación RN5 (-32.827500 ;$56.423889^{\circ}$ ), que cuenta con 24 muestreos de datos de TSA medidos a 300 metros de la costa en Rincón del Bonete obtenidos entre 2010 y 2018. La segunda base corresponde a 43 datos de muestreos en Baygorria desde el 2002 a la fecha (Chalar, et al., 2014, 2015; González-Piana, et al., 2017). Los datos observados (medidos in situ) se graficaron contra los obtenidos del satélite en el punto de Baygorria más cercano a los medidos en campo. Se utilizaron los parámetros de la pendiente e intercepto de la regresión lineal para evaluar la consistencia de las observaciones asumiendo pendiente igual a 1 e intercepto a 0 (Piñeiro, et al., 2008). Como complemento, se calculó la raíz cuadrática del error medio (RMSE) para evaluar la exactitud entre los datos satelitales y los medidos in situ. Cabe destacar que estos muestreos no fueron dirigidos a encontrar sesgos en la medición satelital, sino que son parte de campañas de rutina que mantiene DINAMA, o como parte del convenio UTE-Facultad de Ciencias llevado a cabo en la sección Limnología (Chalar, et al., 2015).

\section{Resultados}

Los resultados se presentan en tres partes. En la primera parte se analizó una única serie temporal del Río Negro promediando los 1513 datos espaciales para cada día. En la segunda parte se trabajó por cada punto de $1 \mathrm{~km}^{2}$ con una serie temporal y los resultados se presentan en forma de mapas. La tercera parte explora la validez de los datos en base al error asociado a la medición remota y al contraste con mediciones in situ. La Figura 1 muestra el mapa del área de estudio con la locación de las grandes represas y algunos centros poblados importantes superpuesto con la temperatura media superficial, la cual es $18,7^{\circ} \mathrm{C}$ aguas arriba y $19,0^{\circ} \mathrm{C}$ aguas abajo.

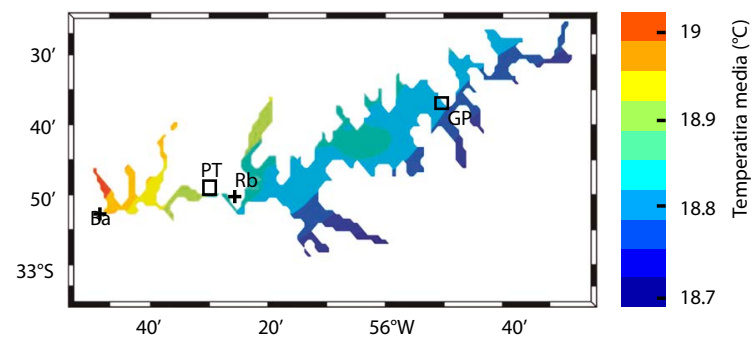

Figura 1. Mapa del área de estudio. En colores se muestra la temperatura media del agua superficial del Río Negro $\left({ }^{\circ} \mathrm{C}\right)$ entre el 1 de junio de 2002 y el 31 de mayo de 2018. Los cuadrados indican la posición de ciudades: San Gregorio de Polanco (GP) y Paso de los Toros (PT). Las cruces indican represas: Rincón del Bonete $(\mathrm{Rb})$ y Baygorria $(\mathrm{Ba})$. 


\section{Ciclo estacional, variabilidad y tendencia del Río Negro promediado espacialmente}

La Figura 2a muestra la serie temporal de TSA utilizada en esta sección, calculada como el promedio espacial de los datos utilizados. Se observa el predominio de la estacionalidad en la variabilidad de la serie temporal (Figura 2a, línea negra). El mínimo de TSA observado fue de $7,80^{\circ} \mathrm{C}$ y sucedió el 2 de agosto de 2007, mientras que el máximo fue de $29,34^{\circ} \mathrm{C}$ el 27 de febrero de 2017. De las anomalías se observa una dominancia de la variabilidad en escala de días, semanas y a nivel estacional, variando entre valores negativos y positivos. Los extremos de anomalías presentan valores cercanos a $-6,00{ }^{\circ} \mathrm{C}$ y $6,00{ }^{\circ} \mathrm{C}$. Estos valores tienen persistencia menor a una semana. Los extremos negativos sucedieron mayormente en setiembre de 2002 y diciembre de 2005 y los positivos en noviembre de 2012. También se observa una tendencia de mayor cantidad de valores positivos hacia el presente. Se destaca la predominancia de la variabilidad estacional que se mantiene aun en las anomalías (Figuras 2 b y $2 c$ ). En cuanto a la cuantificación de las escalas de variabilidad a través del análisis espectral de la serie y las anomalías, se observan tres picos con una periodicidad de 90, 40 y 20 días, de mayor a menor intensidad, asociados con la variabilidad estacional e intraestacional (Figura 2c).
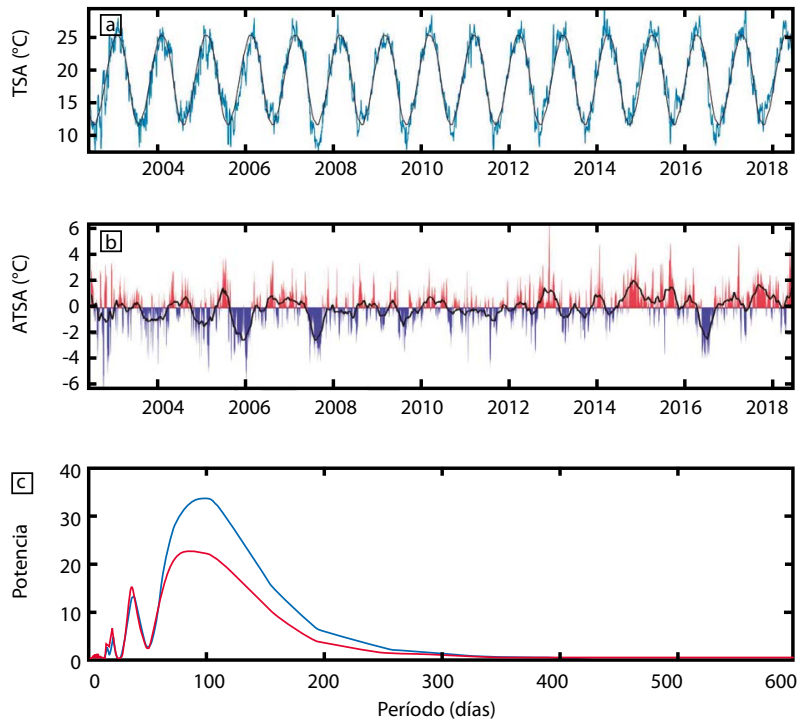

- TSA - ATSA

Figura 2. Análisis de serie temporal de datos diarios de temperatura superficial del agua (TSA) del promedio espacial de los embalses del Río Negro. a) Serie temporal en azul y climatología en negro. b) Anomalías diarias de TSA (ATSA), obtenidas luego de remover la climatología calculada a la serie temporal original; en negro se muestra la media móvil de 90 días de ATSA. c) Análisis espectral de la serie temporal de TSA y ATSA.

La serie temporal promediada espacialmente del Río Negro presenta un ciclo estacional con un promedio anual de $18,83{ }^{\circ} \mathrm{C}$, mientras que el promedio mensual exhibe una mínima de $12,08^{\circ} \mathrm{C}$ y una máxima de $25,23^{\circ} \mathrm{C}$ en julio y enero, respectivamente. El desvío estándar también presenta estacionalidad, con mayor variabilidad durante los meses de invierno, cuando se observa un pico máximo de $1,60^{\circ} \mathrm{C}$ en agosto y un mínimo a fines de marzo de $0,60^{\circ} \mathrm{C}$. También se constatan 3 picos por encima de $1,00^{\circ} \mathrm{C}$ a mediados de junio, principio de noviembre y fines de diciembre en momentos de transición (Figura 3a). En cuanto al análisis de tendencia de la media anual, presenta una correlación de 0,62 (p.valor $<0,05)$ y una tasa de incremento anual (pendiente) de $0,06^{\circ} \mathrm{C}$ por año. Esta tendencia está explicada por las estaciones de primavera y verano, ya que ni invierno ni otoño presentan una tendencia significativa. A su vez, tanto primavera como verano presentan un valor de correlación de 0,71 . Sin embargo, la pendiente para primavera es mayor, $0,14^{\circ} \mathrm{C}$ por año mientras que la de verano es $0,11^{\circ} \mathrm{C}$ por año (Figura $3 b$ ).
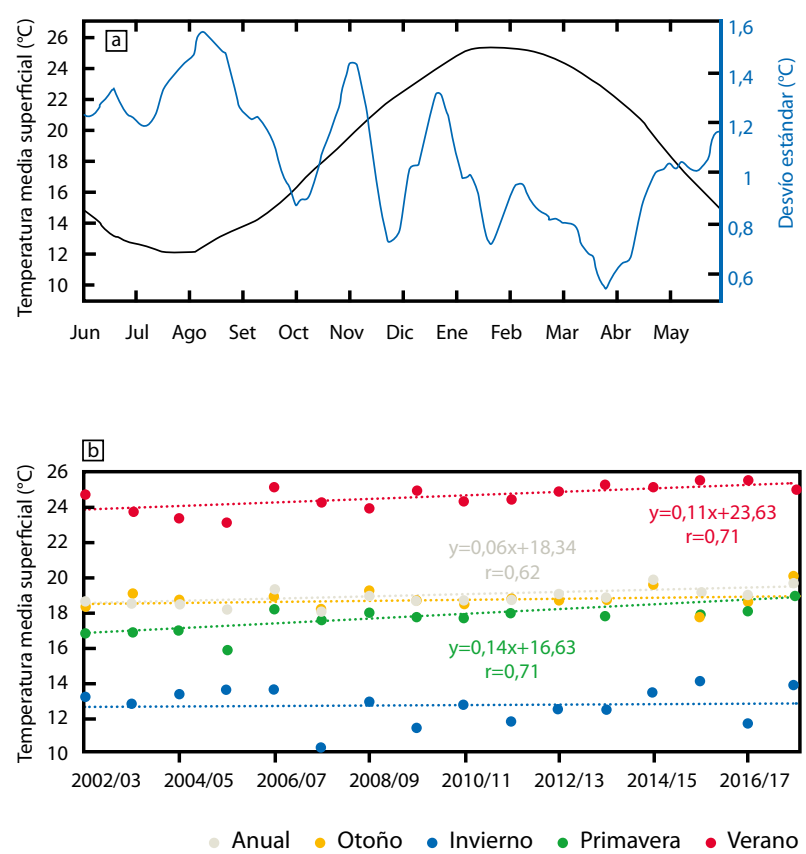

Figura 3. a) Ciclo estacional de temperatura media del agua superficial del Río Negro promediado espacialmente (negro). En azul se muestra el desvío estándar. b) Temperatura media superficial del Río Negro, por año y por estación, en diferentes colores. Cada punto indica la media estacional para cada año. Además, se muestra el ajuste del modelo de regresión lineal, donde la variable explicativa es el tiempo (tendencia lineal). Se muestra la ecuación de la recta (donde "x" es el año desde 2002/03, e "y" es la temperatura predicha por el modelo). También se muestra el coeficiente de correlación de Pearson (r) para aquellos modelos en los que el ajuste es significativo (p.valor $<0,05)$.

\section{Ciclo estacional, variabilidad y tendencia del Río Negro en el espacio}

En el eje espacial se observan ciertas diferencias en el comportamiento de la temperatura superficial del Río Negro, 

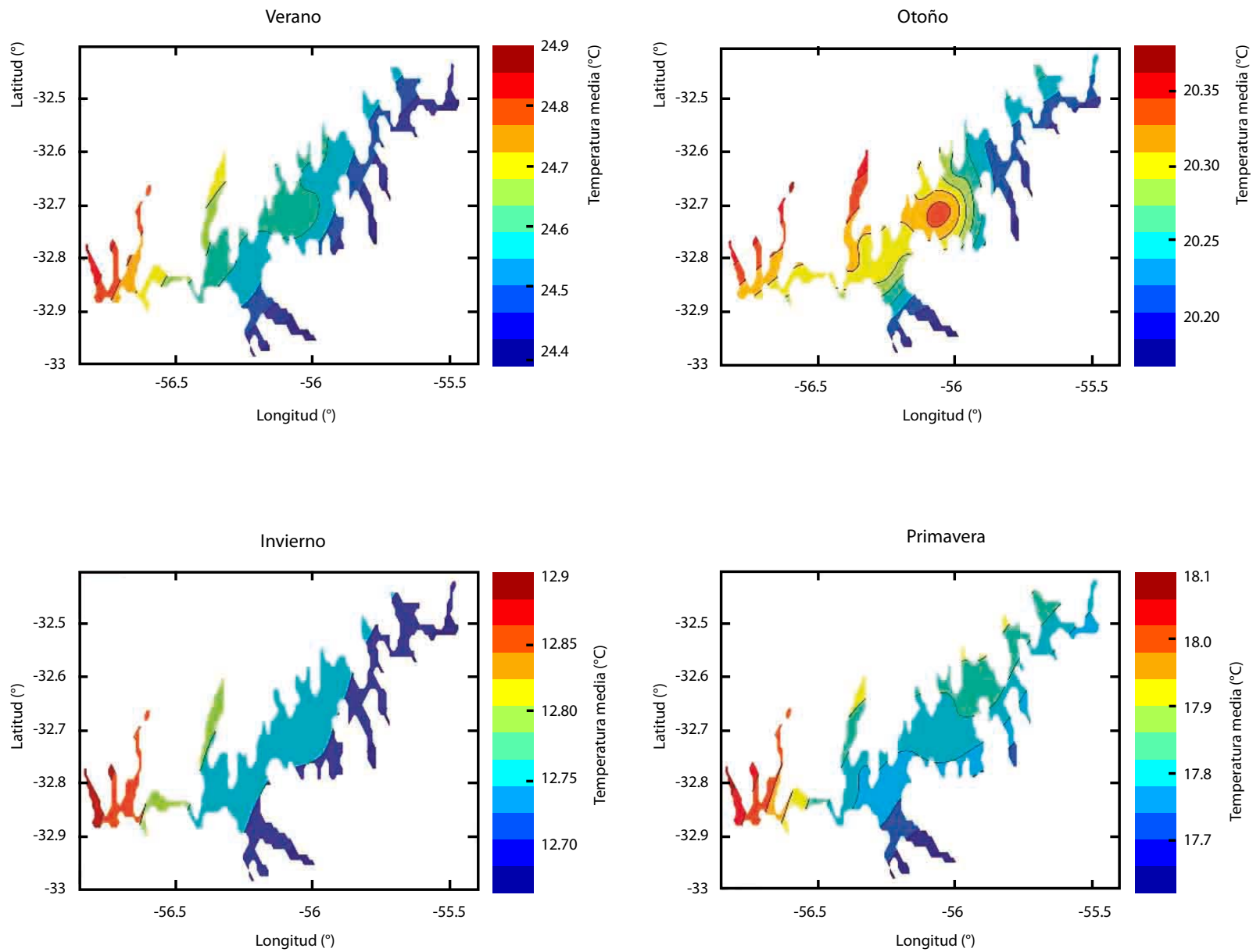

Figura 4. Temperatura media del agua superficial del Río Negro $\left({ }^{\circ} \mathrm{C}\right)$ por estación en el período comprendido entre 1 de junio de 2002 y 31 de mayo de 2018 .

que se analizan a continuación. La media por estación presenta un gradiente en el que la temperatura aumenta desde el Este hacia el Oeste a lo largo de todas las estaciones, y que a su vez crece en amplitud hacia los meses más cálidos, donde la diferencia es de $0,20{ }^{\circ} \mathrm{C}$ en invierno y $0,50{ }^{\circ} \mathrm{C}$ en verano. Invierno presenta una temperatura media de $12,80{ }^{\circ} \mathrm{C}$ y verano de $24,65^{\circ} \mathrm{C}$, respectivamente. En cuanto a las estaciones intermedias, otoño tiende a ser más cálido que primavera $\left(20,30^{\circ} \mathrm{C}\right.$ y $17,90^{\circ} \mathrm{C}$, respectivamente) con una mayor amplitud en el gradiente E-O $\left(0,40{ }^{\circ} \mathrm{C}\right.$ contra $0,30{ }^{\circ} \mathrm{C}$, Figura 4 ).

En cuanto a la variabilidad de la temperatura por estación, se observan patrones distintos en el desvío estándar dentro de cada estación (climatológico) y año a año para cada estación (interanual). En el desvío climatológico se observan los máximos para las estaciones de transición otoño y primavera con valores de $2,96{ }^{\circ} \mathrm{C}$ y $2,76{ }^{\circ} \mathrm{C}$, respectivamente, mientras que invierno y verano presentan valores máximos de $0,78{ }^{\circ} \mathrm{C}$ y $0,88{ }^{\circ} \mathrm{C}$, respectivamente. Otoño y primavera son las estaciones que presentaron mayor variabilidad. A su vez, se observa un patrón en el que primavera-verano tienen mayor variabilidad en la región oeste de la zona de estudio (aguas abajo), y otoñoinvierno la tienen en la región este de la zona de estudio (aguas arriba). La variabilidad interanual tiende a ser menor que la climatológica. Invierno y verano tienen la particularidad de que la variabilidad año a año es mayor que dentro de la estación. Esto se observa especialmente en invierno, cuando el desvío estándar interanual es más del doble que el climatológico, con máximos de $1,75^{\circ} \mathrm{C}$ y $0,78{ }^{\circ} \mathrm{C}$, respectivamente (Figura 5).

Primavera y verano son las únicas estaciones que presentan una tendencia lineal significativa y con pendiente positiva en toda el área de estudio. Para ambas estaciones la tendencia al calentamiento se incrementa hacia el Oeste, y es máxima al Oeste de la ciudad de Paso de los Toros. Sin embargo, la pendiente en primavera es en promedio $0,02^{\circ} \mathrm{C}$. año ${ }^{-1}$ mayor que la de verano. En primavera el mínimo es de $0,13^{\circ} \mathrm{C} . \mathrm{año}^{-1} \mathrm{y}$ el máximo de $0,15^{\circ} \mathrm{C} . \mathrm{anno}^{-1}$, mientras que en verano es $0,10^{\circ} \mathrm{C} . \mathrm{año}^{-1}$ y $0,14^{\circ} \mathrm{C} . \mathrm{año}^{-1}$, respectivamente (Figura 6). El análisis de tendencia para el desvío estándar interanual presenta una pendiente positiva pero no significativa para la media anual y tampoco para las estaciones (no se muestra). 

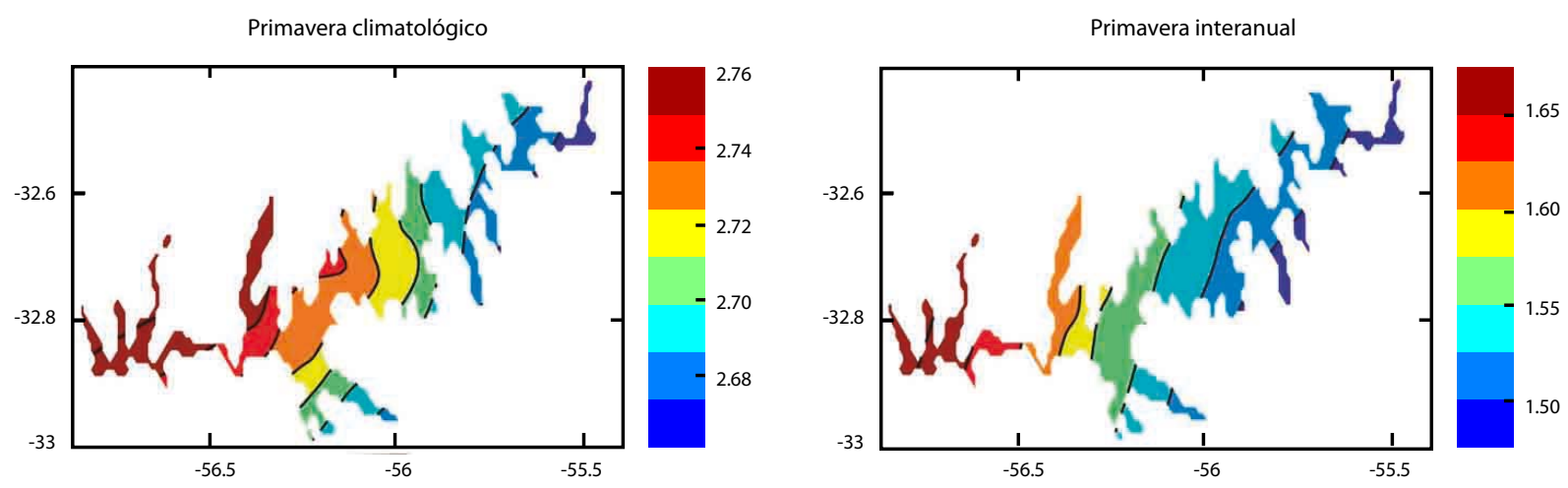

Verano climatológico
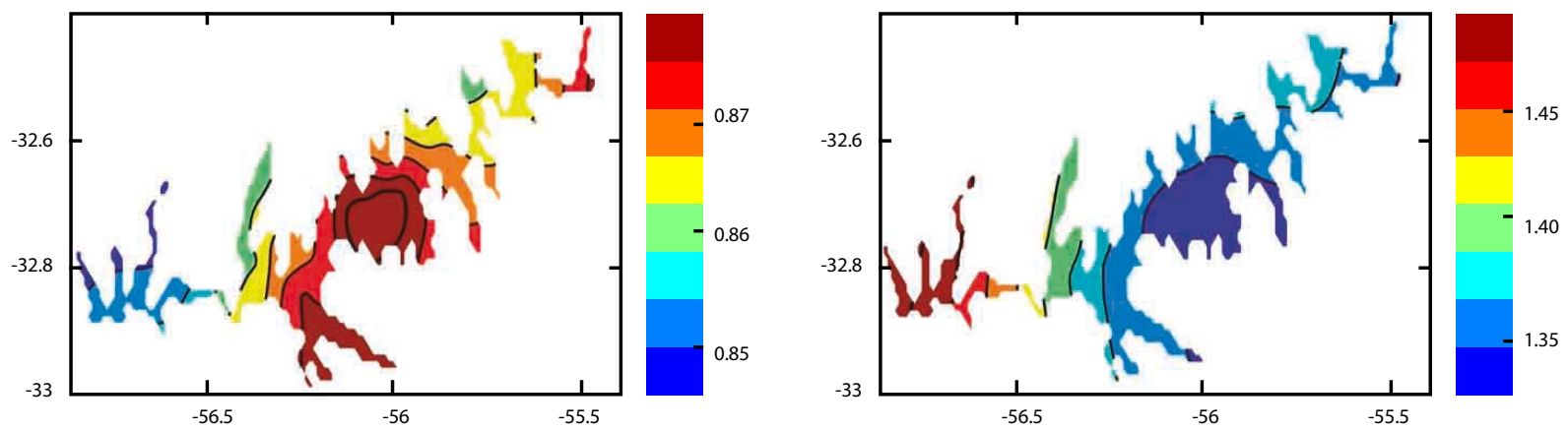

Otoño climatológico

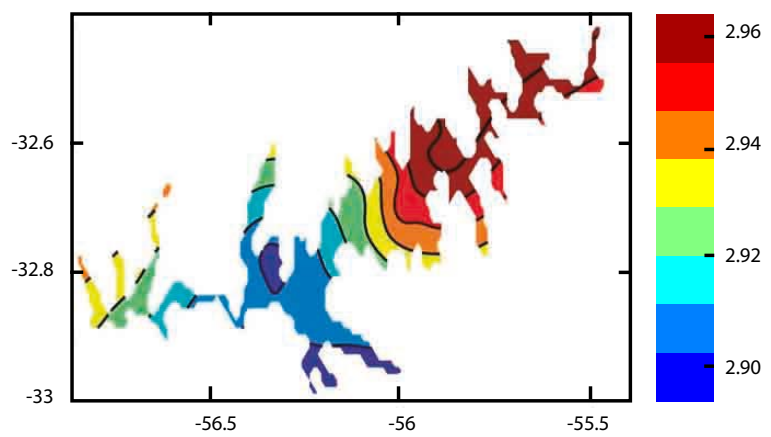

Invierno climatológico
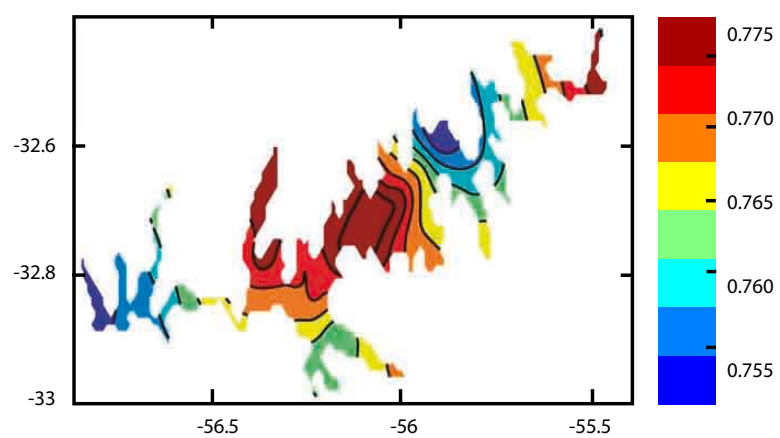

Otoño interanual

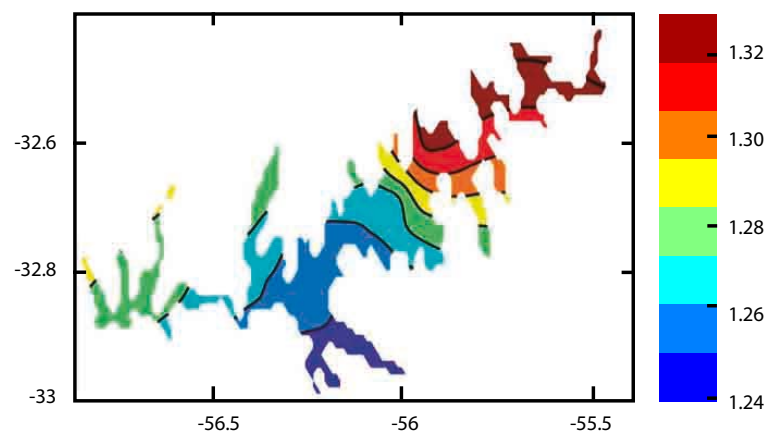

Invierno interanual

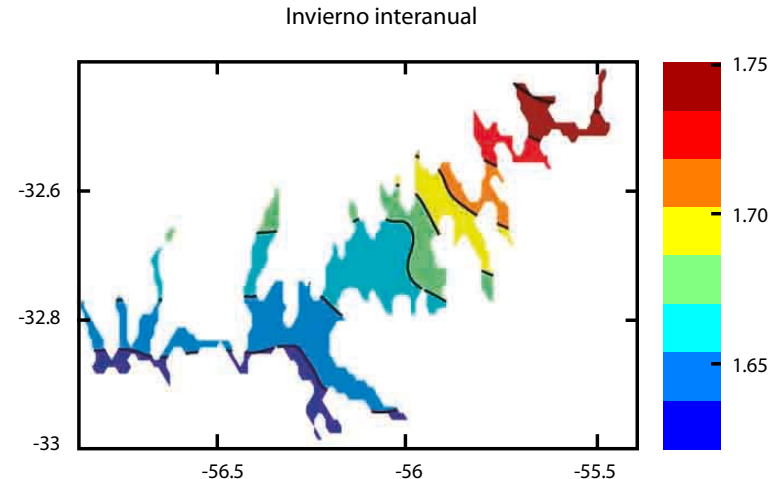

Figura 5. Desvío estándar $\left({ }^{\circ} \mathrm{C}\right)$ climatológico (izquierda) e interanual (derecha) de temperatura superficial en los grandes embalses del Río Negro. Desvío por estación del año a partir de datos diarios en el período comprendido entre 1 de junio de 2002 y 31 de mayo de 2018. Notar las diferencias de escalas. 

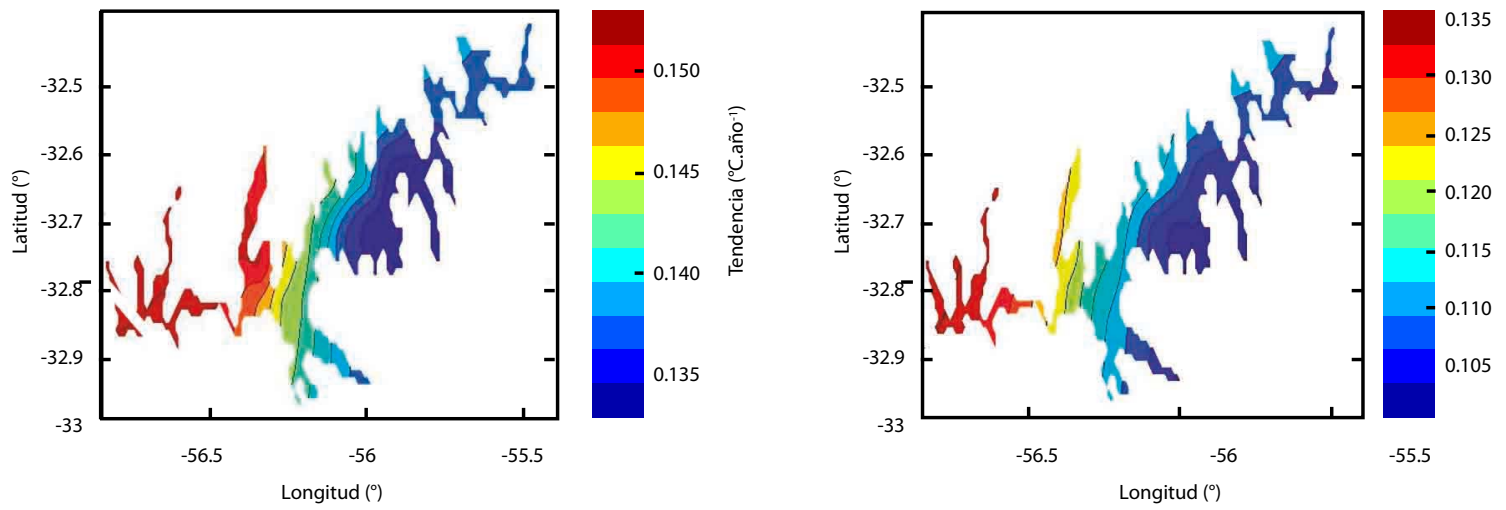

Figura 6. Pendiente para la tendencia $\left({ }^{\circ} \mathrm{C} . a n ̃ o{ }^{-1}\right)$ de temperatura superficial de las aguas de los grandes embalses del Río Negro en primavera (izquierda) y verano (derecha). Solo se muestran las estaciones con tendencia significativa $(\mathrm{p}<0.05)$.
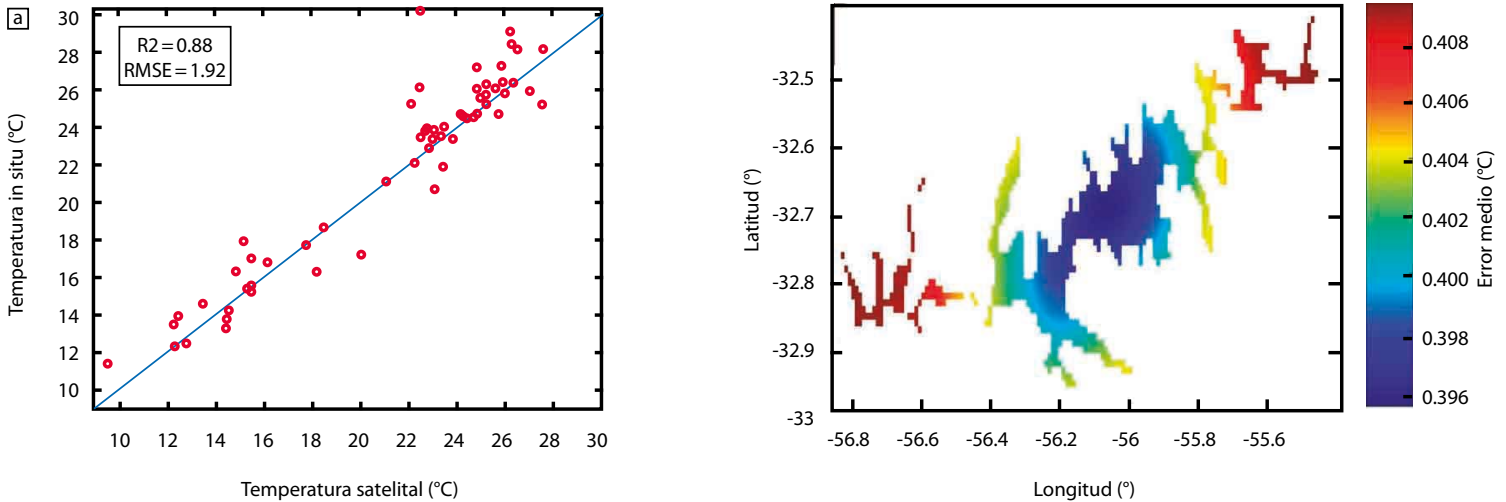

Figura 7. a) Diagrama de puntos entre temperatura satelital y mediciones in situ. Cada punto representa un día de medición en Rincón del Bonete $(n=64)$. En azul se muestra la recta con pendiente 1:1. b) Error medio del dato satelital, promediado en cada punto para los 16 años de datos diarios utilizados.

\section{Comparación de los datos in situ con los satelitales}

El ajuste de la regresión lineal entre ambas fuentes datos presentó una pendiente 0,97 , intercepto igual a 0 , varianza explicada del $88 \%$ y un RMSE de $1,92^{\circ} \mathrm{C}$ (Figura $7 \mathrm{a}$ ). En cuanto al campo espacial del error medio del dato satelital, se observa un patrón concéntrico en el embalse, donde a mayor distancia de la costa, menor error. Los errores máximos se observan en los extremos, aguas arriba y aguas abajo. Sin embargo, las diferencias espaciales son menores a $0,01^{\circ} \mathrm{C}$ en promedio (Figura $7 \mathrm{~b}$ ).

\section{Discusión}

Trabajando con 16 años de datos satelitales (período 20022018) provenientes del producto MUR-GHRSST se encontró una respuesta global de los embalses Baygorria y Rincón de Bonete al aumento de su temperatura en $0,70{ }^{\circ} \mathrm{C}$ por año en la media anual, determinado por una pendiente de incremento de $0,13{ }^{\circ} \mathrm{C}$ por año en primavera-verano. Este aumento, presente principalmente por un calentamiento durante los meses de primavera-verano, podría deberse al calentamiento de la atmósfera reportado para la región (Bidegain, et al., 2014;
IPCC, 2014). Considerando además que se analizaron 16 años de datos, no se descarta que al menos parte de esta tendencia observada podría responder a oscilaciones de baja frecuencia (ej. decadal), mayor al periodo estudiado (Chen y Tung, 2018).

La tendencia al calentamiento encontrada coincide con el rango de valores presentados por O'Reilly et al. (2015) de $0,34^{\circ} \mathrm{C} \cdot$ década $^{-1}\left(\right.$ rango $^{-0,7}$ y $1,3^{\circ} \mathrm{C}$.década $\left.{ }^{-1}\right)$. En ese trabajo, los autores analizan la TSA de verano (obtenida de datos in situ y satelitales) en 235 lagos distribuidos a nivel mundial (periodo 1985 y 2009) y además reportan que coincide con el aumento del promedio anual de la temperatura del aire $\left(0,25^{\circ} \mathrm{C}\right.$. década $\left.^{-1}\right)$ y la temperatura superficial del océano $\left(0,12^{\circ} \mathrm{C}\right.$.década $\left.{ }^{-1}\right)$ durante un período mayor (1979-2012). Dado que los últimos 16 años han sido más cálidos que los anteriores 16 (Smith, et al., 2008), sería esperable que analizando el período tomado por O'Reilly et al. (2015) la pendiente de la tendencia de los embalses del Río Negro fuera menor que la encontrada en este trabajo.

La variabilidad espacial fue menor que la temporal, lo cual es esperable para la extensión de los embalses analizados y su ubicación en latitudes medias. En el eje espacial se encontró un gradiente de aumento de temperatura desde aguas arriba hacia aguas abajo del área estudiada. Este patrón podría relacionarse con los tiempos de residencia del agua, ya que 
el límite de la región aguas abajo se encuentra después del embalse del Rincón del Bonete, cuyo tiempo de residencia es el mayor de los tres con un promedio de 150 días (Chalar, et al., 2014). Al incrementar el tiempo de residencia del agua, aumenta también la exposición a la radiación solar $y$, en consecuencia, al calentamiento durante los meses de primavera-verano (Allan y Castillo, 2007).

Teniendo en cuenta la comparación entre los muestreos in situ y los datos satelitales, los valores de pendiente e intercepto del modelo lineal indican que no hay inconsistencias entre los valores muestreados y los medidos por el satélite (Piñeiro, et al., 2008), y que a su vez el valor de RMSE calculado es esperado para un embalse de este tamaño (Crosman, et al., 2017). Las diferencias encontradas entre los valores in situ y satelitales podrían deberse a que las mediciones in situ son hechas durante el día, mientras que las satelitales se realizan de noche. Para corregir este sesgo de observación, en principio sería necesario conocer la amplitud del ciclo diario de temperatura y corregir los datos. Por otra parte, el hecho de que hacia valores más altos la diferencia sea mayor puede ser explicado por el hecho de que el calentamiento diario en verano es aún mayor. A su vez, los valores muy alejados de la recta de pendiente $1\left(>5^{\circ} \mathrm{C}\right)$ son siempre con mediciones in situ mayores que las satelitales. Esto podría deberse tanto al ciclo diario de temperatura en el embalse, como a mediciones in situ en aguas más cercanas a las costas, donde el ciclo diario tiende a tener aún mayor amplitud debido a que aumenta la relación área' .profundidad ${ }^{-1}$, por lo que sería esperable todavía mayor temperatura en los muestreos durante el día.

De la Figura 7 se puede discutir que a priori no hay razón para determinar que los datos satelitales carecen de utilidad debido a la posible contaminación del dato por la tierra. Y, a su vez, que este set de datos in situ utilizados no es suficiente para identificar sesgos en la medición satelital. Para ello sería necesario contar con una serie temporal de mediciones constantes, de por lo menos varios meses, superficiales, tomadas lejos de la costa y que tomen registros durante la noche (Grim, et al., 2013). De la Figura 7b se desprende que ante una eventual campaña de ajuste de la medición satelital sería recomendable colocar una boya de medición in situ donde el error medio es mínimo, cercana al punto (Lat:-32.70; Lon:-56.05). De esa forma, el producto satelital MUR podría ser utilizado de forma operativa y validado, como se ha demostrado en otros trabajos (Fiedler, et al., 2014; Kraemer, et al., 2017).

En cuanto a las posibles consecuencias ambientales derivadas de los resultados encontrados, bajo condiciones de estratificación térmica asociada al calentamiento de las capas superficiales y baja intensidad de viento se ha observado en Rincón del Bonete una tendencia a la acumulación de cianobacterias potencialmente tóxicas (González-Piana, et al., 2017; González-Piana, et al., 2018). Además, se ha encontrado una relación positiva entre el aumento de la temperatura del agua y la eutrofización con el desarrollo de floraciones en otros embalses y ríos del país: Salto Grande (Martínez de la Escalera, et al., 2017), Río Santa Lucía (Aubriot, et al., 2017) y para todos los sistemas lénticos y lóticos del país considerados en su conjunto (Haakonsson, et al., 2017). Esto se explica porque el aumento de la temperatura produce un aumento de las tasas metabólicas, favoreciendo así a los organismos más pequeños, como los procariotas (Daufresne, et al., 2009; Paerl, 2014). Además, los efectos indirectos de la temperatura, como la estratificación, la reducción de los tamaños corporales de los predadores y la dinámica y disposición de nutrientes son clave para determinar el predominio de las cianobacterias capaces de formar floraciones (Oliver, et al., 2012; Lürling, et al., 2013; Robarts y Zohary, 1987).

En consecuencia, y de acuerdo a la tendencia de calentamiento encontrada durante primavera-verano, este tipo de fenómenos podrían volverse más intensos y recurrentes en este período. Las condiciones favorables podrían asociarse a condiciones sinópticas de viento débil y a aumento de estratificación, con la potencial pérdida de algunos de los servicios ecosistémicos que brindan hoy los grandes embalses del Río Negro.

\section{Conclusiones}

Se caracterizó el ciclo estacional de temperatura superficial de los grandes embalses del Río Negro y su variabilidad a partir de datos satelitales. Estos presentan una media de $18,83^{\circ} \mathrm{C}$ y un promedio mensual con una mínima de $12,08^{\circ} \mathrm{C}$ y una máxima de $25,23{ }^{\circ} \mathrm{C}$ en julio y enero, respectivamente. Se observó una tendencia significativa de aumento de temperatura superficial del Río Negro en primavera-verano de $1,3{ }^{\circ} \mathrm{C}$ por década, no así en los meses de otoño e invierno que presentan una mayor variabilidad interanual. Existe un patrón espacial para primavera-verano en el que la temperatura y la tendencia al calentamiento aumentan desde aguas arriba hacia aguas abajo. Dado el estado eutrófico del Río Negro, sería recomendable contemplar un escenario de primaveras y veranos más cálidos en el manejo ambiental. La herramienta aquí presentada sirve como insumo para los actuales planes de monitoreo del río, y eventual modelación con variables biológicas, con la ventaja de su inmediatez a un costo prácticamente nulo, aunque debe ser validada con mediciones in situ dirigidas. A su vez, los resultados hallados sirven de insumo para las proyecciones del plan de manejo del Río Negro y sus posibles consecuencias ambientales.

\section{Reconocimientos}

Al Group for High Resolution Sea Surface Temperature (GHRSST) y DINAMA por la libre disponibilidad de los datos. También al Dr. Guillermo Chalar y su grupo de investigación (Sección Limnología, IECA, Facultad de Ciencias, UdelaR) y el convenio UTE- Fcien, por compartir la base de datos de temperatura in situ del embalse del Rincón del Bonete. A los revisores que colaboraron con la mejora sustancial del artículo.

\section{Referencias}

Allan, J.D. y Castillo, M.M., 2007. Stream ecology: structure and function of running waters. Berlín: Springer Science \& Business Media.

Aubriot, L., Delbene, L., Haakonsson, S., Somma, A., Hirsch, F. y Bonilla, S., 2017. Evolución de la eutrofización en el Río Santa Lucía: influencia de la intensificación productiva y perspectivas. En: INNOTEC, 14, pp.7-16.

Barton, I.J., 1995. Satellite-derived sea surface temperatures: current status. En: Journal of Geophysical Research: Oceans, 100(C5), pp.8777-8790.

Bidegain, M., Crisci, C., del Puerto, L., Inda, H., Mazzeo, N., Taks, J. y Terra, R., 2014. Clima de cambios: nuevos desafíos de adaptación en Uruguay. Volumen 1. Variabilidad climática de importancia para el sector productivo [En línea]. Montevideo: FAO, MGAP. [Consulta: 20 de 
setiembre de 2018]. Disponible en: http://www.fao.org/ docrep/field/009/as253s/as253s.pdf

Bonilla, S., Haakonsson, S., Somma, A., Gravier, A., Britos, A., Vidal, L. y de la Escalera, G.M., 2015. Cianobacterias y cianotoxinas en ecosistemas límnicos de Uruguay. En: INNOTEC, (10), pp.9-22.

Brown, J.H., Gillooly, J.F., Allen, A.P., Savage, V.M. y West, G.B., 2004. Toward a metabolic theory of ecology. En: Ecology, 85(7), pp.1771-1789.

Chalar, G., Fabián, D., González-Piana, M. y Piccardo, A., 2015. Estado y evolución de la calidad de agua de los tres embalses del Río Negro. Montevideo: Facultad de Ciencias; UTE.

Chalar, G., Gerhard, M., González-Piana, M. y Fabián, D., 2014. Hidroquímica y eutrofización en tres embalses subtropicales en cadena. En: Marcovecchio, J.E., Botté, S.E. y Freije, R.H. Procesos geoquímicos superficiales en Sudamérica. Salamanca: Nueva Graficesa. pp.121-148.

Chang, N. B., Imen, S. y Vannah, B., 2015. Remote sensing for monitoring surface water quality status and ecosystem state in relation to the nutrient cycle: A 40-year perspective. En: Critical Reviews in Environmental Science and Technology, 45(2), pp.101-166.

Chen, X. y Tung, K.K., 2018. Global surface warming enhanced by weak Atlantic overturning circulation. En: Nature, 559(7714), pp.387.

Chin, Toshio M., Milliff, Ralph F. y Large, William G., 1998. Basin-scale, high-wavenumber sea surface wind fields from a multiresolution analysis of scatterometer data. En: Journal of atmospheric and oceanic technology, 15(3), pp.741-763.

Crosman, E., Vazquez-Cuervo, J. y Chin, T., 2017. Evaluation of the multi-scale ultra-high resolution (MUR) analysis of lake surface temperature. En: Remote Sensing, 9(7), pp.723.

Dash, P., Ignatov, A., Martin, M., Donlon, C., Brasnett, B., Reynolds, R. W. y Grumbine, R., 2012. Group for high resolution sea surface temperature (GHRSST) analysis fields inter-comparisons-Part 2: Near real time webbased level 4 SST Quality Monitor (L4-SQUAM). En: Drinkwater, Kenneth, Arístegui, Javier, eds. Deep sea research part II: Topical studies in oceanography, 77, pp.31-43.

Daufresne, M., Lengfellner, K. y Sommer, U., 2009. Global warming benefits the small in aquatic ecosystems. En: Proceedings of the National Academy of Sciences, 106(31), pp.12788-12793.

DINAMA, 2018. OAN observatorio ambiental nacional. Módulo calidad de agua. (Base de datos 12/09/2017). [En línea]. Montevideo: DINAMA. [Consulta: 20 de setiembre de 2018]. Disponible en: https://www.dinama.gub.uy/oan.

Fiedler, Emma K., Martin, Matthew J. y Roberts-Jones, Jonah, 2014. An operational analysis of lake surface water temperature. En: Tellus A: Dynamic Meteorology and Oceanography, 66(1), pp.21247.

González-Piana, M., Fabian, D., Delbene, L. y Chalar, G., 2011. Toxics blooms of Microcystis aeruginosa in three Rio Negro reservoirs, Uruguay. En: Harmful Algae News, 43, pp.16-17.

González-Piana, M., Fabián, D., Piccardo, A. y Chalar, G., 2017. Dynamics of total Microcystin LR concentration in three subtropical hydroelectric generation reservoirs in Uruguay, South América. En: Bulletin of Environmental Contamination and Toxicology, 99(4), pp.488-492.

González-Piana, M., Piccardo, A., Ferrer, C., Brena, B., Pírez, M., Fabián, D. y Chalar, G., 2018. Effects of wind mixing in a stratified water column on toxic cyanobacteria and
Microcystin-LR distribution in a subtropical reservoir. En: Bulletin of environmental contamination and toxicology, pp.1-6.

Grim, Joseph A., Knievel, Jason C. y Crosman, Erik T., 2013. Techniques for using MODIS data to remotely sense lake water surface temperatures. En: Journal of Atmospheric and Oceanic Technology, 30(10), pp.2434-2451.

Haakonsson, S., Rodríguez-Gallego, L., Somma, A. y Bonilla, S., 2017. Temperature and precipitation shape the distribution of harmful cyanobacteria in subtropical lotic and lentic ecosystems. En: Science of the Total Environment, 609, pp.1132-1139.

Hambright, K.D., Gophen, M. y Serruya, S., 1994. Influence of long-term climatic changes on the stratification of a subtropical, warm monomictic lake. En: Limnology and Oceanography, 39(5), pp.1233-1242.

Hutchinson, G.E., 1941. Limnological studies in Connecticut: IV. The mechanisms of intermediary metabolism in stratified lakes. En: Ecological Monographs, 11(1), pp.21-60.

IPCC, 2014. Climate change 2013 - The physical science basis: Working Group I contribution to the fifth assessment report of the intergovernmental panel on climate change. Cambridge: Cambridge University Press. doi:10.1017/ СВО9781107415324

Joehnk, K.D., Huisman, J.E.F., Sharples, J., Sommeijer, B.E.N., Visser, P.M. y Stroom, J.M., 2008. Summer heatwaves promote blooms of harmful cyanobacteria. En: Global Change Biology, 14(3), pp.495-512.

Kraemer, B. M., Mehner, T. y Adrian, R., 2017. Reconciling the opposing effects of warming on phytoplankton biomass in 188 large lakes. En: Scientific Reports, 7(1), pp.10762.

Lürling, M., Eshetu, F., Faassen, E.J., Kosten, S. y Huszar, V.L., 2013. Comparison of cyanobacterial and green algal growth rates at different temperatures. En: Freshwater Biology, 58(3), pp.552-559.

Margalef, R., 1983. Limnología. Vol. 1009. Barcelona: Omega.

Martin, M., Dash, P., Ignatov, A., Banzon, V., Beggs, H., Brasnett, B. y Grumbine, R., 2012. Group for High Resolution Sea Surface temperature (GHRSST) analysis fields inter-comparisons. Part 1: A GHRSST multi-product ensemble (GMPE). En: Deep Sea Research Part II: Topical Studies in Oceanography, 77, pp.21-30.

Martínez de la Escalera, G., Kruk, C., Segura, A. M., Nogueira, L., Alcántara, I. y Piccini, C., 2017. Dynamics of toxic genotypes of Microcystis aeruginosa complex (MAC) through a wide freshwater to marine environmental gradient. En: Harmful Algae, 62, pp.73-83.

MGAP, 2017. Anuario estadístico DIEA 2017 [En línea]. Montevideo: MGAP. [Consulta: 28 de junio 2018]. Disponible en: http://www.mgap.gub.uy/sites/default/files/ diea-anuario2017web01a.pdf

MVOTMA, 2018. Indicadores ambientales. Concentración de fósforo total (PT) [En línea]. Montevideo: MVOTMA. [Consulta: 28 de junio de 2018]. Disponible en:

https://www.dinama.gub.uy/indicadores_ambientales/ficha/ oan-concentracion-de-fosforo-total/

Oliver, R.L., Hamilton, D.P., Brookes, J.D. y Ganf, G.G., 2012. Physiology, blooms and prediction of planktonic cyanobacteria. En: Whitton B., ed. Ecology of cyanobacteria II. Dordrecht: Springer. pp.155-194.

O'Reilly, C. M., Sharma, S., Gray, D. K., Hampton, S. E., Read, J. S., Rowley, R. J. y Weyhenmeyer, G. A., 2015. Rapid and highly variable warming of lake surface waters around the globe. En: Geophysical Research Letters, 42(24), pp.10-773. 
Paerl, H.W., 2014. Mitigating harmful cyanobacterial blooms in a human-and climatically-impacted world. En: Life, 4(4), pp.988-1012.

Pérez, M.D.C., 2002. Fitoplancton del río Negro, Uruguay. En: Limnetica, 21(1-2), pp.81-92.

Piñeiro, G., Perelman, S., Guerschman, J. P. y Paruelo, J. M., 2008. How to evaluate models: observed vs. predicted or predicted vs. observed? En: Ecological Modelling, 216(3-4), pp.316-322.

Poole, G.C. y Berman, C.H., 2001. An ecological perspective on in-stream temperature: natural heat dynamics and mechanisms of human-caused thermal degradation. En: Environmental Management, 27(6), pp.787-802.

Press, W.H., Teukolsky, S.A., Vetterling, W.T. y Flannery, B.P., 1992. Numerical recipes. Cambridge: Cambridge University Press.

Reynolds, C.S., 2006. The ecology of phytoplankton. Cambridge: Cambridge University Press.

Robarts, R.D. y Zohary, T., 1987. Temperature effects on photosynthetic capacity, respiration, and growth rates of bloom-forming cyanobacteria. En: New Zealand Journal of Marine and Freshwater Research, 21(3), pp.391-399.
Sharma, S., Gray, D. K., Read, J. S., O'Reilly, C. M., Schneider, P., Qudrat, A. y Lenters, J. D., 2015. A global database of lake surface temperatures collected by in situ and satellite methods from 1985-2009. En: Scientific Data, 2, pp.150008.

Smith, T.M., Reynolds, R.W., Peterson, T.C. y Lawrimore, J., 2008: Improvements to NOAA's historical merged landocean surface temperatures analysis (1880-2006). En: Journal of Climate, 21, pp.2283-2296.

Straile, D., 2005. Food webs in lakes-seasonal dynamics and the impact of climate variability. En: Belgrano, A., Sharler, U., Dunne, J.y Ulanowicz, R., eds. Aquatic food webs. An ecosystem approach. En: New York: Oxford University Press. pp.41-50.

Tundisi, J.G., Matsumura-Tundisi, T., Arantes Junior, J.D., Tundisi, J. E.M., Manzini, N. F. y Ducrot, R., 2004. The response of Carlos Botelho (Lobo, Broa) reservoir to the passage of cold fronts as reflected by physical, chemical, and biological variables. En: Brazilian Journal of Biology, 64(1), pp.177-186.

Webb, B.W., Hannah, D.M., Moore, R.D., Brown, L.E. y Nobilis, F., 2008. Recent advances in stream and river temperature research. En: Hydrological Processes, 22(7), pp.902-918. 